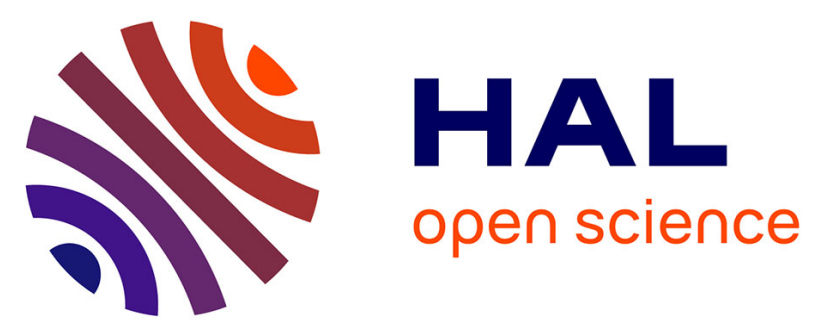

\title{
INGESTION SPONTANÉE D'ÉLÉMENTS ÉNERGÉTIQUES EN RÉGIMES MIXTES ET SÉPARÉS CHEZ LE RAT EN CROISSANCE
}

Y. Henry, A. Rérat, Yvonne Daniel, M. Lecourtier

\section{- To cite this version:}

Y. Henry, A. Rérat, Yvonne Daniel, M. Lecourtier. INGESTION SPONTANÉE D'ÉLÉMENTS ÉNERGÉTIQUES EN RÉGIMES MIXTES ET SÉPARÉS CHEZ LE RAT EN CROISSANCE. Annales de biologie animale, biochimie, biophysique, 1965, 5 (2), pp.283-292. hal-00896289

\section{HAL Id: hal-00896289 \\ https://hal.science/hal-00896289}

Submitted on 1 Jan 1965

HAL is a multi-disciplinary open access archive for the deposit and dissemination of scientific research documents, whether they are published or not. The documents may come from teaching and research institutions in France or abroad, or from public or private research centers.
L'archive ouverte pluridisciplinaire HAL, est destinée au dépôt et à la diffusion de documents scientifiques de niveau recherche, publiés ou non, émanant des établissements d'enseignement et de recherche français ou étrangers, des laboratoires publics ou privés. 
Ann. Biol. anim. Bioch. Biophys., I965, 5 (2), 283-292.

\title{
INGESTION SPONTANÉE D'ÉLÉMENTS ÉNERGÉTIQUES EN RÉGIMES MIXTES ET SÉPARÉS CHEZ LE RAT EN CROISSANCE
}

\author{
Y. HENRY et A. RÉRAT \\ Avec la collaboration technique de Yvonne Daniel et M. Lfcourtier \\ Station de Recherches sur l'Élevage des Porcs, \\ Centre national de Recherches zootechniques, Jouy-en-Josas (Seine-et-Oise)
}

SOMMAIRE

L'étude de la consommation spontanée d'énergie a été réalisée chez le rat en croissance, selon qu'on lui administre en mélange et à volonté un régime semi-synthétique, ou en deux repas séparés, une quantité équivalente de la même source azotée et de l'énergie à volonté. Deux expériences ont ainsi été effectuées, d'une durée de 4 et 8 semaines, au cours desquelles la source azotée était constituée soit par une farine de hareng, soit par un tourteau d'arachide.

Lorsque le Rat est placé en dessous des conditions de croissance maximum, l'administration séparée des éléments azotés et énergétiques provoque, par rapport à l'alimentation mixte, une réduction sensible de la consommation spontanée d'énergie. La légère dépression de la croissance qui en résulte se traduit essentiellement par une diminution du dépôt de lipides dans les tissus, la rétention azotée n'étant pratiquement pas modifiée.

\section{IN'TRODUCTION}

Au cours d'études antérieures (HENRy et RÉRAT, I962; RÉRAT et HENRY, I963), il a été constaté que le Rat, lorsqu'il reçoit séparément et simultanément des substances azotées en quantité limitée et des éléments énergétiques à volonté, ajuste sa consommation d'énergie en fonction du niveau de croissance permis par la fraction azotée du régime. Il a, en outre, tendance à maintenir une composition corporelle constante, à un poids donné, quelle que soit la nature de la source azotée. Or, on sait que dans le cas des régimes complets habituels, la composition corporelle, pour un niveau de croissance déterminé, varie selon la nature de la source azotée (Al,ISON, I957). D'autre part, si l'on en juge d'après les résultats acquis en régime 
mixte dans d'autres expériences, les Rats soumis à l'alimentation séparée semblent présenter une adiposité plus faible que ceux recevant des régimes mixtes pour un niveau de croissance comparable. Afin de vérifier cette observation, nous avons entrepris une expérimentation sur des couples de Rats soumis en même temps à 1'alimentation séparée ou mixte et recevant quotidiennement la même quantité de matières azotées, la nature de ces dernières et leur niveau dans la ration étant variable.

\section{TECHNIQUES EXPÉRIMENTALES ETT MATÉRIEL UTILISÉ}

Deux expériences successives A et B sont réalisées sur deux groupes de 80 et 75 rats mâles de souche Wistar $C$. F., soumis pendant une semaine à un régime préexpérimental à base de caséine dont la composition a été rapportée par ailleurs (HENRY et RÉRAT, i962).

TABLEAU I

Schéma expérimental

\begin{tabular}{|c|c|c|c|c|}
\hline Expérience & \multicolumn{2}{|c|}{$\mathrm{A}$} & & \\
\hline Nombre d'animaux par lot & \multicolumn{2}{|c|}{6} & & \\
\hline Nature de la source azotée & \multicolumn{2}{|c|}{ F. de poisson } & $\mathrm{T}$. & chide \\
\hline Durée (semaines) & 4 & 8 & & \\
\hline Protéines p. 100 & 12 & 12 & 8 & 16 \\
\hline Régime mixte & \multicolumn{4}{|c|}{ ad libitum } \\
\hline $\begin{array}{l}\text { Régines séparés : } \\
\text { — repas azoté } \\
\text { — repas non azoté }\end{array}$ & \multicolumn{4}{|c|}{$\begin{array}{c}\text { ajusté sur celui du partenaire en mixte } \\
\text { ad libilum }\end{array}$} \\
\hline
\end{tabular}

Schéma de l'analyse de variance

\begin{tabular}{|c|c|c|c|}
\hline Expérience A & $\mathrm{DL}$ & Expérience B & $\mathrm{DL}$ \\
\hline $\begin{array}{l}\text { Effet couples } \ldots \ldots \ldots \ldots \\
\text { Age } \ldots \ldots \ldots \\
\text { Niveau azoté }(\mathrm{N}) \ldots \ldots \ldots \ldots \\
\mathrm{N} \times \text { âge } \ldots \ldots \ldots \ldots \ldots \\
\text { Erreur }(a) \ldots \ldots \ldots \ldots \ldots \\
\text { Mode d'alimentation mixte- } \\
\quad \text { séparé }(\mathrm{R}) \ldots \ldots \ldots \ldots \ldots \\
\mathrm{R} \times \text { âge } \ldots \ldots \ldots \ldots \ldots \\
\mathrm{R} \times \mathrm{N} \ldots \ldots \\
\mathrm{R} \times \mathrm{N} \times \text { âge } \ldots \ldots \ldots \ldots \\
\text { Erreur }(b) \ldots \ldots \ldots\end{array}$ & $\begin{array}{r}23 \\
1 \\
1 \\
1 \\
20 \\
\\
1 \\
1 \\
1 \\
1 \\
20\end{array}$ & $\begin{array}{l}\text { Effet couples } \ldots \ldots \ldots \ldots \\
\mathrm{N} \ldots \ldots \ldots \ldots \\
\text { Erreur }(a) \ldots \ldots \ldots \ldots \\
\mathrm{R} \ldots \ldots \ldots \ldots \ldots \\
\mathrm{R} \times \mathrm{N} \ldots \ldots \ldots \ldots \ldots\end{array}$ & $\begin{array}{r}25 \\
1 \\
24 \\
1 \\
1 \\
24\end{array}$ \\
\hline Variation totale $\ldots \ldots \ldots$ & 47 & Variation totale $\ldots \ldots \ldots$ & 51 \\
\hline
\end{tabular}


Dans chacune des deux expériences, les animaux sont répartis par couples homogènes, d'après leur poids, leur gain de poids et leur consommation pendant la période préexpérimentale. Un animal de chaque couple reçoit ad libitum un régime mixte renfermant soit de la farine de poisson de Norvège (expérience $\mathrm{A}$ ), soit du tourteau d'arachide (expérience $\mathrm{B}$ ), ̀̀ deux taux différents, respectivement 8 et i 2 p. roo de matières azotèes dans l'expérience $A, 8$ et I6 p. roo dans l'expérience B. Au deuxième animal, on distribue quotidiennement, dans deux mangeoires séparées, un repas azoté apportant la même quantité d'azote que celle ingérée la veille par son partenaire, et un repas non azoté ad libitum. Le premier jour, le régime mixte est offert aux deux animaux dans les mêmes conditions. Les deux sources azotées ont été choisies en raison de leur valeur biologique très différente et sont introduites dans le régime à des taux inférieurs à ceux correspondant à la croissance maximum, ces derniers étant respectivement i6 et $24 \mathrm{p}$. Ioo pour la farine de poisson et le tourteau d'arachide (RÉRAT et HENRY, résultats non publiés). Afin de rendre les régimes comparables, tant du point de vue de l'appétibilité que du point de vue de la valeur énergétique, on ajoute dans tous les cas des quantités constantes d'huile d'arachide (8 p. roo), de cellulose ( 2 p. I00), de minéraux (3 p. I00) et de vitamines ( 2 p. I00).

TABLEAU 2

Composition des régimes

\begin{tabular}{|c|c|c|c|c|c|c|c|c|}
\hline \multirow{4}{*}{$\begin{array}{l}\text { Expériences } \ldots \ldots \ldots \ldots \ldots \cdots \cdots \cdots \\
\text { Régimes } \quad \ldots \ldots \ldots \ldots \ldots \ldots \ldots \cdots \cdots\end{array}$} & \multicolumn{4}{|c|}{ A } & \multicolumn{4}{|c|}{ B } \\
\hline & \multirow{2}{*}{\multicolumn{2}{|c|}{ Mixtes }} & \multicolumn{2}{|c|}{ Séparés } & \multirow{2}{*}{\multicolumn{2}{|c|}{ Mixtes }} & \multicolumn{2}{|c|}{ Séparés } \\
\hline & & & azoté & non & & & azoté & non \\
\hline & 8 & 12 & 36 & - & 8 & 16 & 43 & - \\
\hline 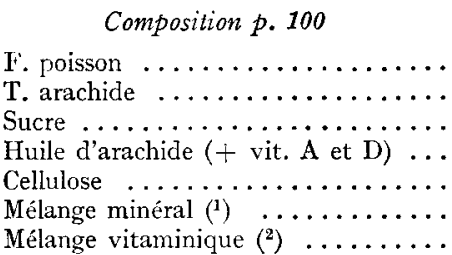 & $\begin{array}{r}11 \\
75 \\
8 \\
2 \\
3 \\
1\end{array}$ & $\begin{array}{l}16,5 \\
\frac{-}{69,5} \\
8 \\
2 \\
3 \\
1\end{array}$ & $\begin{array}{r}50 \\
- \\
36 \\
8 \\
2 \\
3 \\
1\end{array}$ & $\begin{array}{l}- \\
- \\
86 \\
8 \\
2 \\
3 \\
1\end{array}$ & $\begin{array}{r}- \\
15 \\
71 \\
8 \\
2 \\
3 \\
1\end{array}$ & $\begin{array}{l}- \\
30 \\
56 \\
8 \\
2 \\
3 \\
1\end{array}$ & $\begin{array}{c}\overline{81,4} \\
4,6 \\
8 \\
2 \\
3 \\
1\end{array}$ & $\begin{array}{r}- \\
- \\
86 \\
8 \\
2 \\
3 \\
1\end{array}$ \\
\hline $\begin{array}{l}\quad \text { Résultats d'analyse } \\
\text { N p. } 100 \text { mat. sèche }: \ldots \ldots \ldots \ldots \\
\text { Kcal/g mat. sèche } \vdots \\
\text { • valeur mesurée à la bombe } \\
\text { calorimétrique adiabatique } \ldots \ldots \ldots \\
\text { - Valeur estimée }\left({ }^{3}\right) \ldots \ldots \ldots \ldots \ldots\end{array}$ & $\overline{4,34}$ & $\overline{4,41}$ & $\overline{4,85}$ & $\overrightarrow{4,18}$ & $\begin{array}{l}4,36 \\
4,43\end{array}$ & $\begin{array}{l}4,57 \\
4,53\end{array}$ & $\begin{array}{l}4,91 \\
4,89\end{array}$ & $\begin{array}{l}4,36 \\
4,36\end{array}$ \\
\hline
\end{tabular}

(1) Selon Osborne et Mendel (1918).

(2) Henry et Rerat, (1963).

(3) D'après les coefficients d'Atwater et Bryant (1903).

La durée de l'expérience est de 4 semaines (expérience A) ou de 8 semaines (expérience A et B). Les schémas expérimentaux et la composition des régimes sont rapportés respectivement dans les tableaux I et 2. Huit lots de 6 rats ont ainsi été constitués dans la première expérience et 4 lots de I 3 Rats dans la seconde. Les animaux, élevés en cages individuelles, sont pesés à intervalles réguliers (deux fois par semaine) et les consommations de nourriture sont enregistrées quoditiennement. A la fin de l'expérience on procède à l'analyse corporelle; les rétentions azotée et énergétique sont déterminées suivant la méthode exposée précédemment (HENRY et RéRAT, I962).

Les teneurs en énergie des régimes et des carcasses ont été estimées d'après les coefficients 
calorimétriques d'Atwater et Bryant (1903). De plus, dans l'expérience B, il a été procédé à une détermination directe de la valeur énergétique des régimes par combustion dans une bombe calorimétrique adiabatique. Les chiffres ainsi obtenus sont très voisins des estimations théoriques (tabl. 2); ceci justifie la méthode de calcul employée, d'autant plus que la même concordance a été observée dans le cas des carcasses.

Le dispositif expérimental, du type factoriel $2^{3}$ dans l'expérience $\mathrm{A}$ et $2^{2}$ dans l'expérience $B$, a été conçu suivant la méthode de superposition de l'effet blocs et d'un effet principal (niveau azoté) : "split-plot" avec "confounding" (Cochran et Cox, 1957). Au cours de l'analyse statistique des résultats, les effets principaux et interactions ont ainsi été testés par rapport à l'une ou l'autre de deux variances résiduelles : une erreur interblocs $(a)$ et une erreur intra-blocs (b) (tabl. I). Afin de faciliter la lecture des tableaux de résultats, seuls ont été mentionnés les effets qui ont atteint le seuil de signification 0,05 ou 0,01 , ainsi que la valeur de l'écart-type de la moyenne, déduite de l'erreur individuelle $(b)$.

\section{RÉSULTATS}

\section{Croissance et consommation (tableaux 3 et 4 )}

Comme cela était prévu par le protocole, les animaux soumis à l'alimentation séparée ont ingéré sensiblement la même quantité d'azote, après 4 ou 8 semaines, que ceux recevant les régimes mixtes correspondants; il en a été de même pendant toute la durée de l'expérience (fig. I). Dans ces conditions, l'administration séparée des repas azoté et non azoté entraîne par rapport à l'alimentation mixte liabituelle :

- une diminution de la vitesse de croissance, qui n'est toutefois significative que dans le cas du tourteau d'arachide (expérience B),

- une réduction significative de la consommation d'énergie.

Cette différence dans le niveau de croissance et la consommation d'énergie se maintient d'ailleurs tout au long de l'expérience, comme l'indique la figure I.

Si l'on considère les variations de la réponse en fonction du niveau azoté, on relève dans le cas de l'arachide une interaction significative entre le mode d'alimentation (mixte-séparé) et le niveau azoté ; les différences entre les deux types de régime sont alors plus marquées au niveau azoté supérieur ( $15 \mathrm{p}$. Ioo de protéines) qu'au bas niveau ( 8 p. Ioo de protéines). Cette interaction n'existe pas pour la farine de poisson ; cependant, il faut noter que les deux niveaux azotés sont beaucoup plus rapprochés dans ce cas que dans celui de l'arachide.

\section{Utilisation de l'azote et de l'énergie}

Les critères d'utilisation de l'azote (coefficient d'efficacité protidique, coefficient d'utilisation pratique de l'azote) et de l'énergie (indice de consommation, coefficient d'utilisation pratique de l'énergie) ne sont pas modifiés d'une manière significative par le mode d'apport des éléments azotés et énergétiques.

En ce qui concerne le niveau azoté, des différences apparaissent selon la nature de la source azotée et l'âge des animaux. Ainsi, avec la farine de poissonl, l'efficacité est maximum pour I 2 p. Ioo de protéines jusqu'à 4 semaines et pour 8 p. Ioo seulement au-delà de 4 semaines; avec le tourteau d'arachide, au contraire, les valeurs observées sont plus faibles et augmentent avec le taux de protéines dans les limites considérées. Quant au coefficient d'utilisation pratique de l'énergie, il est pratiquement le même pour les deux niveaux de protéines de poisson, tandis qu'il augmente avec le niveau de protéines d'arachide. 


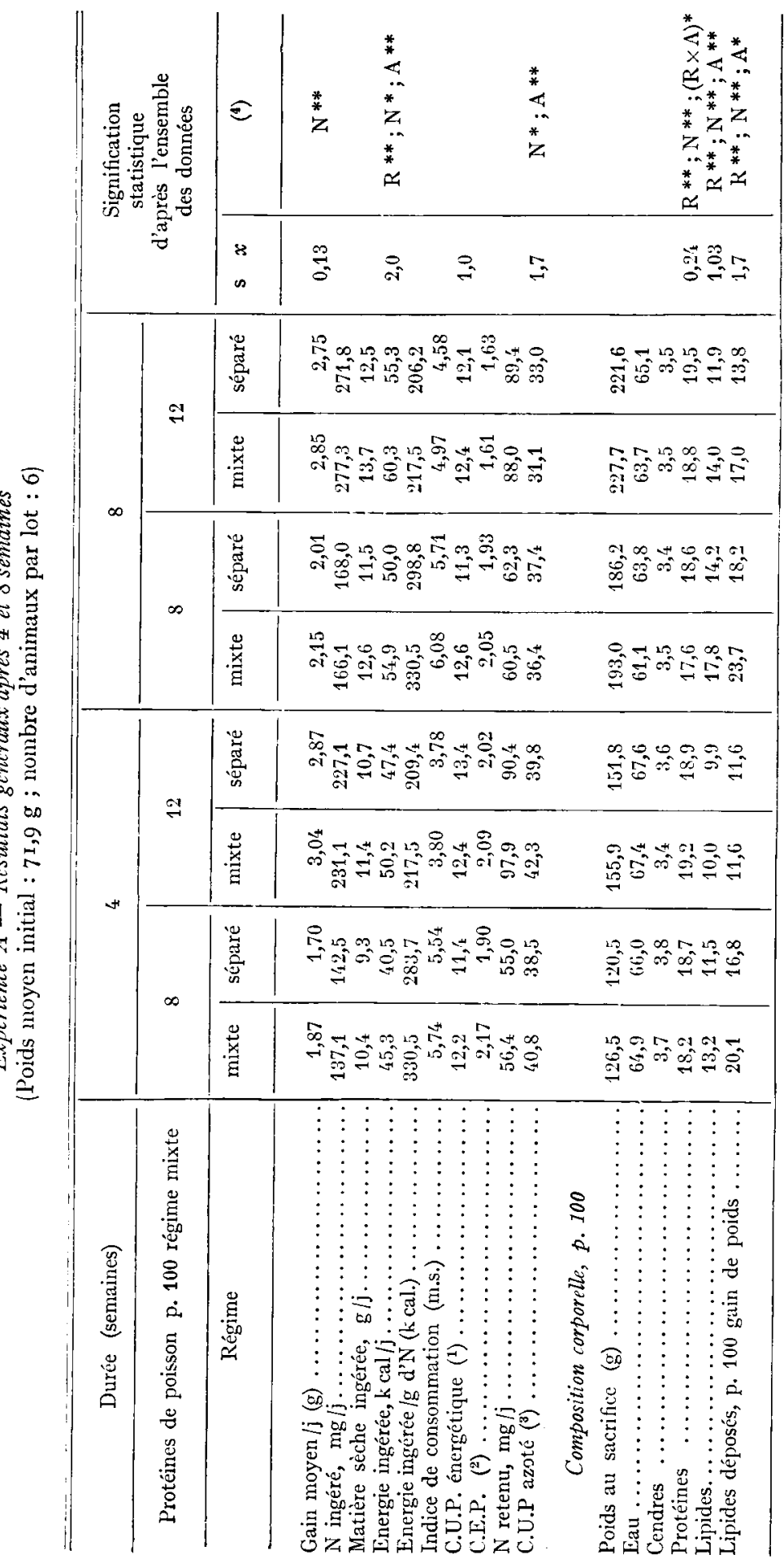

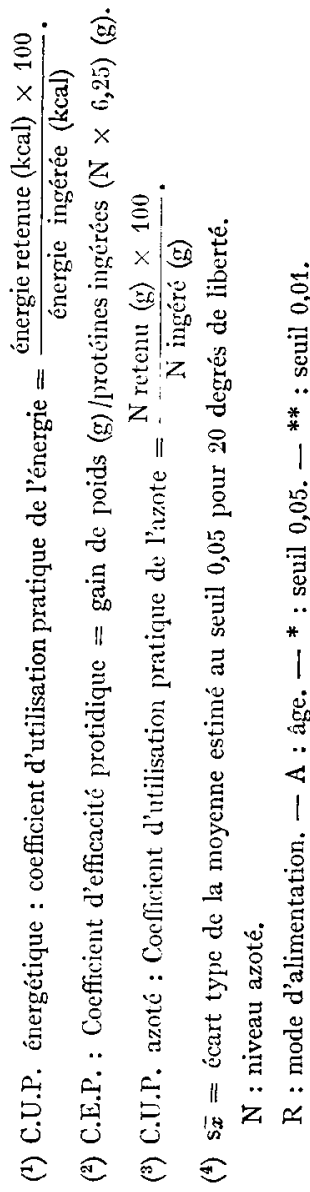



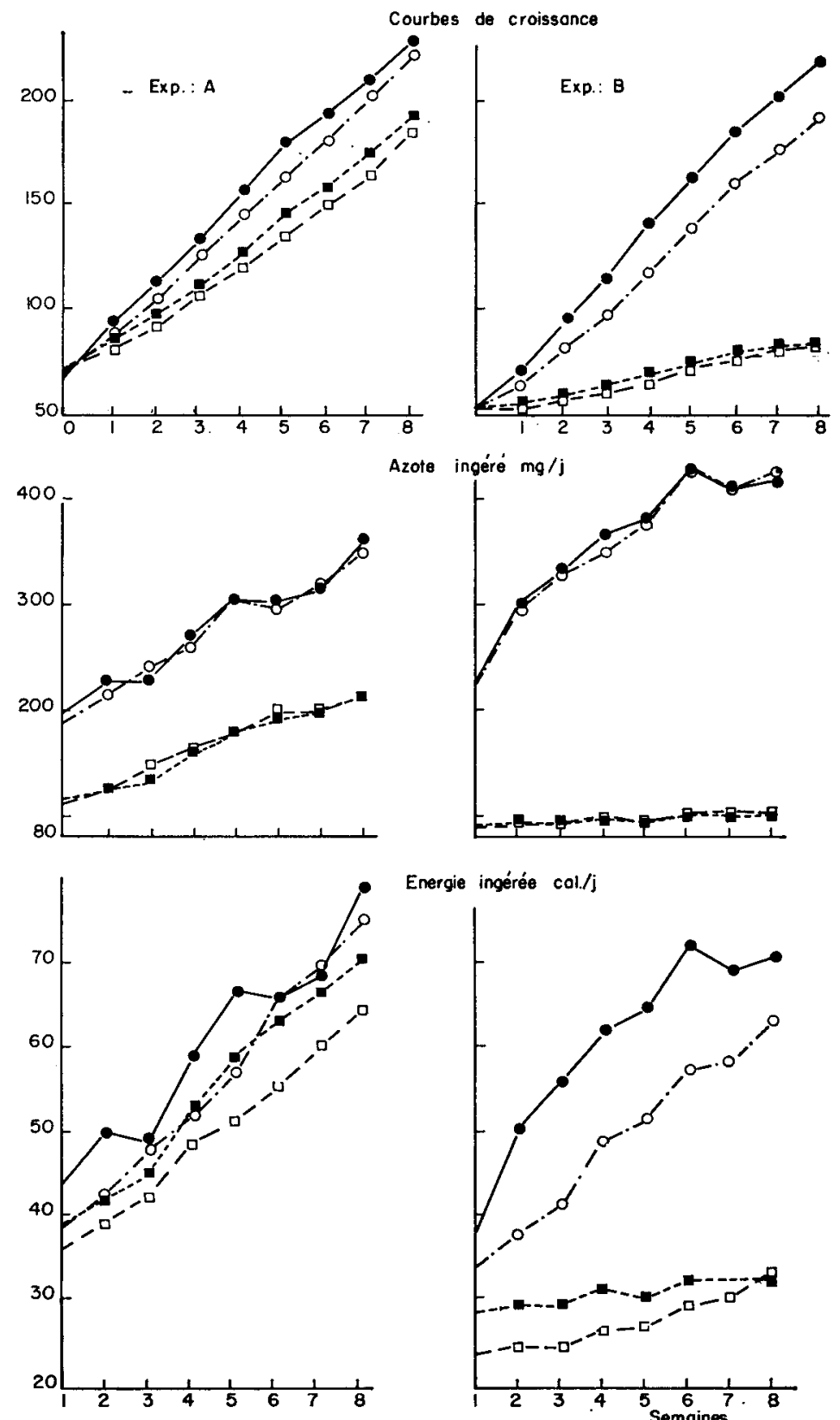

Energie ingérée col./j
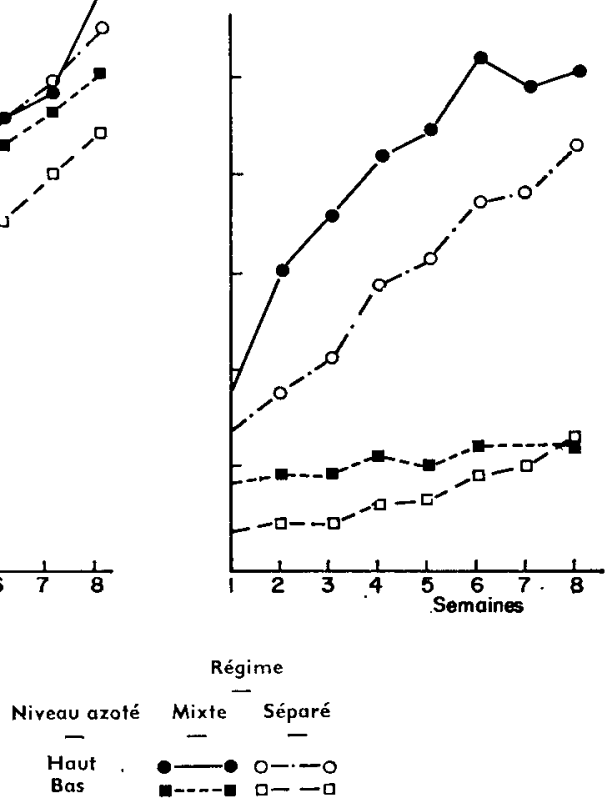

FIG. I. - Evolution des critères au cours de la croissance 


\section{Composition corporelle}

Tant sur la proportion de protéines que sur celle de lipides dans la carcasse, on note une influence hautement significative du niveau azoté et du mode d'alimentation, sans qu'il y ait interaction entre ces deux facteurs. Ainsi, il se produit une augmentation du pourcentage de protéines trouvées dans les tissus, lorsqu'on

TABLEAU 4

Expérience B: Tourteau d'arachide - Résultats généraux après 8 semaines (poids moyen initial : $54,2 \mathrm{~g}$; nombre d'animaux par lot : $\mathrm{I}_{3}$ )

\begin{tabular}{|c|c|c|c|c|c|c|}
\hline \multirow{2}{*}{$\begin{array}{l}\text { Protéines d'arachicle, p. } 100 \\
\text { (régime mixte.) }\end{array}$} & \multicolumn{2}{|c|}{8} & \multicolumn{2}{|c|}{16} & \multicolumn{2}{|c|}{$\begin{array}{c}\text { Signification } \\
\text { statistique } \\
\text { d'après l'ensemble } \\
\text { des données }\end{array}$} \\
\hline & mixte & séparé & mixte & séparé & $s_{\bar{x}}\left(^{4}\right)$ & \\
\hline Gain moyen $/ \mathrm{j}(\mathrm{g}) \ldots \ldots$ & 0,61 & 0,55 & 2,95 & 2,48 & 0,05 & $\mathrm{R}^{* *} ; \mathrm{N}^{* *} ;(\mathrm{R} \times \mathrm{N})^{* *}$ \\
\hline $\mathrm{N}$ ingéré, $\mathrm{mg} / \mathrm{j} \ldots \ldots \ldots$ & 98,9 & 97,9 & 359,5 & 352,1 & & \\
\hline Mat. sèche ingérée, $g / \mathrm{j} \ldots$ & 7,32 & 6,45 & 13,31 & 10,73 & & \\
\hline Énergie ingérée, kcal $/ j \ldots \ldots \ldots$. & 31,9 & 28,8 & 60,8 & 49,4 & 1,2 & $\mathrm{R}^{* *} ; \mathrm{N}^{* *} ;(\mathrm{R} \times \mathrm{N})^{* *}$ \\
\hline Énergie ingérée, par g d'N (kcal).... & 323,0 & 300,0 & 169,2 & 140,7 & & \\
\hline Indice de consommation (mat. sèche). & $13,0^{\prime}$ & 16,07 & 4,55 & 4,36 & & \\
\hline C.U.P. énergétique $(1) \ldots \ldots \ldots \ldots$ & 5,4 & 5,5 & 14,0 & 12,8 & 0,4 & $\mathrm{~N}^{* *}$ \\
\hline C.E.P, $\left({ }^{2}\right) \ldots \ldots \ldots \ldots$ & 0,96 & 0,88 & 1,33 & 1,13 & & \\
\hline $\mathrm{N}$ retenu, $\mathrm{mg} / \mathrm{j} \ldots \ldots \ldots \ldots \ldots$ & 18,0 & 18,4 & 89,5 & 79,3 & & \\
\hline C.U.P. azoté $(\mathbf{3}) \ldots \ldots \ldots \ldots \ldots$ & 17,8 & 18,7 & 24,8 & 22,5 & 0,9 & $\mathrm{~N}^{* *}$ \\
\hline Composition corporelle, $p .100$ & & & & & & \\
\hline Poids au sacrifice $(g) \ldots \ldots \ldots \ldots \ldots$ & 88,9 & 85,3 & 219,6 & 192,3 & & \\
\hline $\operatorname{Ea} \ldots \ldots \ldots \ldots \ldots \ldots \ldots \ldots \ldots$ & 65,1 & 65,8 & 61,7 & 64,2 & & \\
\hline Cendres................... & 4,1 & 4,2 & 3,0 & 3,3 & & \\
\hline Protéines $\ldots \ldots \ldots \ldots \ldots \ldots \ldots \ldots \ldots$ & 18,5 & 19,3 & 19,0 & 19,7 & 0,1 & $\mathrm{R}^{* *} ; \mathrm{N}^{* *}$ \\
\hline Lipides $\ldots \ldots \ldots \ldots \ldots \ldots \ldots \ldots$ & 12,3 & 10,7 & 16,3 & 12,8 & 0,5 & $\mathrm{R}^{* *} ; \mathrm{N}^{* *}$ \\
\hline Lipides déposés P. 100 de gain de poids. & 22,2 & 20,8 & 19.3 & 15,2 & 1,3 & $\mathrm{R}^{*} ; \mathrm{N}^{* *}$ \\
\hline
\end{tabular}

(1) C.U.P. énergétique : coefficient d'utilisation pratique de l'énergie $=\frac{\text { énergie retenue (kcal) }}{\text { énergie ingérée }(k c a l)} \times 100$.

$\left.{ }^{2}\right)$ C.E.P. : Coefficient d'efficacitẻ protidique $=$ gain de poids $(\mathrm{g}) /$ protéines ingérées $(\mathrm{N} \times 6,25)(\mathrm{g})$.

$\left({ }^{3}\right)$ C.L.P. azoté : coefficient d'utilisation pratique de l'azote $=\frac{N \text { retenu }(\mathrm{g})}{\mathrm{N} \text { ingéré }(\mathrm{g})} \times 100$.

(4) $s_{\bar{x}}=$ écart-type de la moyenne estimé au seuil 0,05 pour 20 degrés de liberté.

$\mathrm{R}$ : mode d'alimentation. $-\mathrm{A}$ : âge. $-\mathrm{N}$ : niveau azoté.

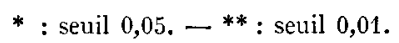

augmente le niveau azoté, mais ce phénomène est imputable à l'augmentation de poids des animaux en fin d'expérience (RÉRA'T et HENRY, I963). I1 n'en est pas de même pour le mode d'alimentation : à poids final pratiquement égal, l'administration de repas séparés provoque une réduction de l'adiposité et plus précisément un abaissement du pourcentage de lipides dans le gain du poids (fig. 2).

Annales de Biologie animale. - 1965 . 

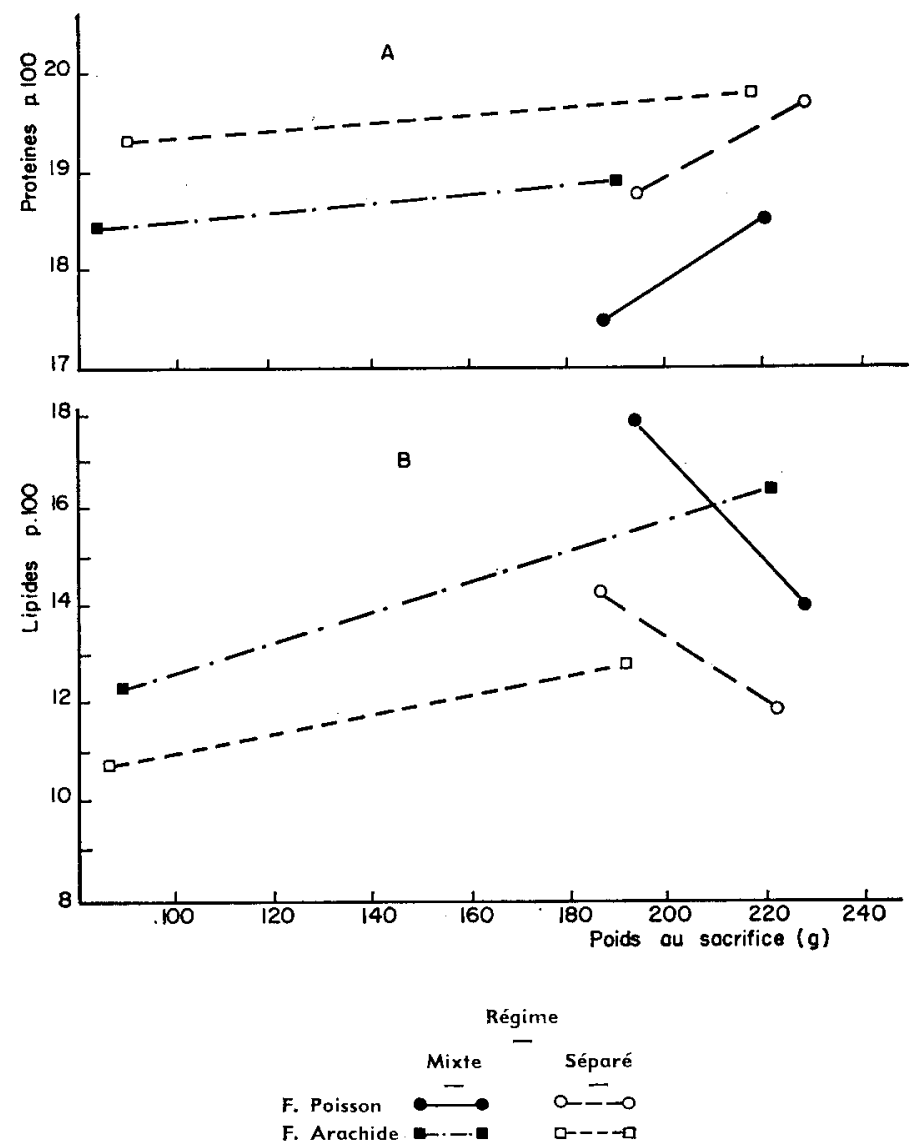

FIG. 2. - Influence du mode d'alimentation sur la composition corporelle (après 8 semaines)

A. Pourcentage des protéines dans la carcasse

B. Pourcentage de lipides dans la carcasse

\section{DISCUSSION}

D'après les résultats précédents, il apparaît que le Rat, en alimentation séparée, présente une vitesse de croissance légèrement plus faible qu'en alimentation mixte. A cet égard, il convient tout d'abord de remarquer que les écarts de croissance enregistrés sont dus pour une part aux conditions expérimentales. Ainsi, une période d'une semaine au départ de l'expérience a été nécessaire pour que les animaux s'habituent à la distribution de repas séparés, alors que les régimes mixtes ont été consommés sans difficulté dès le début. Ceci explique le fait que les différences dans les gains de poids sont relativement plus importantes après 4 semaines qu'après 8 semaines d'expérience. D'autre part, les rats en alimentation séparée ne se sont pas trouvés 
tout à fait dans les conditions d'alimentation ad libitum, puisque leur ingestion d'azote a été réglée sur celle des animaux des lots " mixtes " correspondants ; dans ces conditions, ils n'ont pu, comme ces derniers, extérioriser pleinement leurs potentialités de croissance. C'est du reste ce qui différencie cette expérience de celle effectuée par CALET et al. (I96I) sur le poulet, où les deux régimes, mixte et séparé, étaient restreints quant à 1'apport azoté. L'écart dans les gains de poids peut être partiellement attribué à ces phénomènes.

Quoi qu'il en soit, la dépression de croissance consécutive à l'administration de repas séparés est toujours liée à une réduction importante de la consommation d'énergie. Comme les animaux ainsi traités utilisent l'azote et l'énergie avec la même efficacité qu'en régime mixte, ceci se traduit au niveau des tissus par une diminution sensible du dépôt de lipides. Cette différence dans le comportement des animanx selon le mode d'apport des éléments azotés et énergétiques peut s'expliquer. On sait, en effet, que dans les deux expériences, les animaux sont placés en dessous des conditions de croissance maximum; celle-ci, dans le cas de la farine de poisson, ne pourrait être atteinte qu'avec un régime renfermant I6 p. Ioo de protéines et serait de l'ordre de $5,0 \mathrm{~g} / \mathrm{j}$ pendant 8 semaines après le sevrage; dans le cas $\mathrm{du}$ tourteau d'arachide, les données correspondantes seraient $24 \mathrm{p}$. Ioo et $4,4 \mathrm{~g} / \mathrm{j}$ (RÉRAT et HENRY, résultats non publiés). On peut dès lors penser que les animaux recevant ces régimes mixtes à apport insuffisant de protéines, réagissent en tentant d'en ingérer le plus possible; ils consommeraient ainsi un excès de matière sèche et, par suite, d'énergie qui se dépose sous forme de lipides dans les tissus. Par contre, les animaux soumis à l'alimentation séparée et pour lesquels l'apport azoté est également insuffisant ont la possibilité, du fait du mode d'alimentation, de régler leur consommation énergétique en fonction du niveau de croissance permis par le facteur limitant; il n'y a plus hyperconsommation d'énergie comme dans le cas précédent et les animaux déposent moins de lipides dans leurs tissus. Cette explication est valable pour chacun des niveaux azotés considérés et pour chacun des aliments azotés utilisés dans nos expériences. I1 semble toutefois qu'elle doive être nuancée dans le cas des animaux recevant de l'arachide au plus faible niveau azoté : l'apport azoté quantitatif et qualitatif n'est alors qu'en léger excédent par rapport au besoin d'entretien qui prend une part prépondérante dans le besoin global de croissance; il est alors possible que le phénomène d'hyperconsommation du régime mixte s'atténue, ce qui annule pratiquement les différences entre les deux types de régime. Par ailleurs, il serait intéressant de connaître ce qui se passe lorsque le besoin azoté pour une croissance maximum est couvert : selon les hypothèses émises précédemment, on peut penser que le comportement des rats serait le même en alimentation mixte et en alimentation séparée.

En résumé, lorsque le Rat reçoit, séparément, un repas azoté en quantité limitée, inférieure à celle correspondant à la croissance maximum, et un repas non azoté ad libitum, il ingère spontanément moins d'énergie et présente une vitesse de croissance plus lente que lorsqu'on lui offre ad libitum le même repas azoté sous forme de mélange. La légère dépression de la croissance consécutive à 1'administration de repas séparés provient en réalité d'un dépôt plus faible de lipides dans les tissus, la rétention azotée étant aussi élevée qu'en régime mixte. 


\section{SUMMARY}

\section{VOLUNTARY INTAKE OF ENERGY BY GROWING RATS GIVEN MIXED FEEDS OR THE SEPARATE COMPONENTS.}

During two experiments with growing white rats, lasting 4 and 8 weeks, a study was made of voluntary intake of energy according to whether the feed was mixed or the components given separately, both supplying the same amount of protein daily.

Male weanling Wistar C. F. rats were divided into similar pairs at the start of a pre-experimental period of one week. Within each pair one animal was given to appetite a mixed diet with, in the first experiment (A) 8 or $\mathrm{I} 2$ per cent fish protein, and in the second (B) 8 or 16 per cent groundnut protein. The other member of the pair was given daily, in two separate feeders, an amount of the same protein equivalent to the amount eaten by the pair-mate the day before, and a protein-free feed to appetite.

In all cases the animals given the separate feeds grew more slowly than those receiving the same protein in a mixed feed. However, the difference in growth only reached significance on the larger amount of groundnut protein. The small depression in growth when the separate feeds were given was accompanied by a marked reduction in intake of energy compared with that on the usual mixed feed. As a result, in the tissues there was a marked reduction in deposition of lipids and an increase in the protein of the carcass, while the efficiency of utilization of energy and of protein was not changed.

It may be concluded that when rats are given separately a protein feed restricted to less than the amount which would allow maximum growth, and a non protein feed to appetite, they adjust their intakes of energy to a level below that in a mixed feed supplying the same intake of protein.

\section{RÉFÉRENCES BIBLIOGRAPHIQUES}

Allison J. B., 1957. Calories and protein nutrition. Ann. N. Y., Acad. Sci., 69, Ioog-1or8.

Atwater W. O., Bryant A. P., rgo3. The chemical composition of american food materials. $U . S$. Dept. Agric. Bull., 28.

Calet C., Jouandet C., Baratou J., ig6r. Variation de la consommation spontanée d'énergie du poussin en fonction de la nature des matières azotées du régime. Ann. Biol. anim. Bioch. Biophys., 1, 5-9.

Cochran W. G., Cox G. M., I957. Experimental designs, Pp 611., J. Wiley and Sons, New-York.

HENRY Y., Rérat A., 1962. Influence de la qualité et de la quantité des matières azotées ingérées sur la consommation spontanée d'énergie chez le rat en croissance. Ann. Biol. anim. Bioch. Biophys., 2, $267-276$.

Osborne T. B., Mendel L. B., I9r8. The inorganic elements in Nutrition. J. Biol. Chem., 34, I3I-I40.

Rérat A., HEnry Y., I963. Étude de l'ajustement de la consommation spontanée d'énergie en fonction de l'apport azoté chez le rat en croissance. Ann. Biol. anim. Bioch. Biophys., 3, 263-298. 